Q

Check for

updates

Cite as

Nano-Micro Lett.

(2019) 11:85

Published online: 15 October 2019

(C) The Author(s) 2019,

corrected publication 2019

\section{Correction to: Fluorescent Silicon Nanorods-Based Nanotheranostic Agents for Multimodal Imaging-Guided Photothermal Therapy}

\author{
Mingyue Cui ${ }^{1}$, Sangmo Liu ${ }^{1}$, Bin Song ${ }^{1}$, Daoxia Guo ${ }^{1}$, Jinhua Wang ${ }^{1}$, Guyue $\mathrm{Hu}^{1}$, \\ Yuanyuan $\mathrm{Su}^{1 凶}$, Yao $\mathrm{He}^{1 凶}$ \\ The original article can be found online at https://doi.org/10.1007/s40820-019-0306-9. \\ $\triangle$ Yuanyuan Su, suyuanyuan@suda.edu.cn; Yao He, yaohe@suda.edu.cn \\ 1 Laboratory of Nanoscale Biochemical Analysis Institute of Functional Nano \& Soft Materials (FUNSOM), \\ and Jiangsu Key Laboratory for Carbon-Based Functional Materials \& Devices, Soochow University, 199 \\ Ren'ai Road, Suzhou 215123, Jiangsu, People's Republic of China
}

\section{Correction to: Nano-Micro Lett. (2019) 11:73 https://doi.org/10.1007/s40820-019-0306-9}

In the original publication figures $3 \mathrm{~b}, 5 \mathrm{a}, 5 \mathrm{~g}$ are incorrectly published and the scale bars in figures $4 \mathrm{~b}$ and $4 \mathrm{~d}$ have not appeared.

The correct figures are provided in this correction.

The original article has been corrected.
Open Access This article is distributed under the terms of the Creative Commons Attribution 4.0 International License (http://creat ivecommons.org/licenses/by/4.0/), which permits unrestricted use, distribution, and reproduction in any medium, provided you give appropriate credit to the original author(s) and the source, provide a link to the Creative Commons license, and indicate if changes were made. The Creative Commons Public Domain Dedication waiver (http://creativecommons.org/publicdomain/zero/1.0/) applies to the data made available in this article, unless otherwise stated. 
(a)

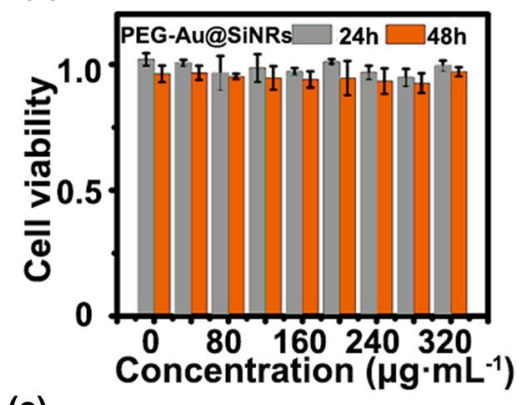

(c)

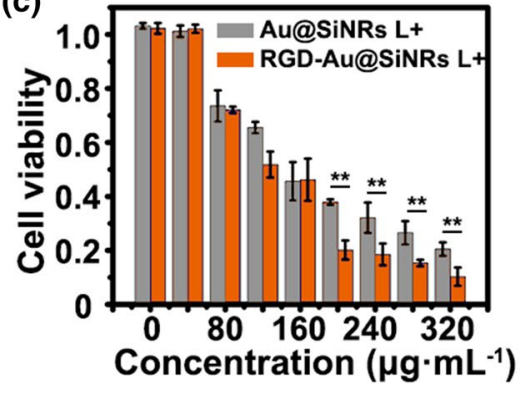

(b)

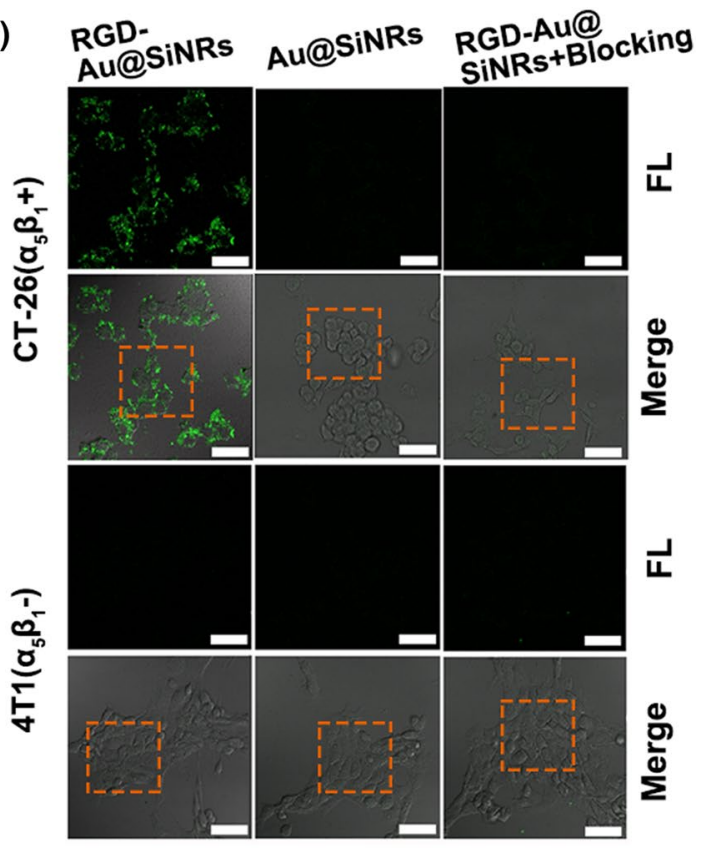

Fig. 3 Assessment of the biocompatibility, targeted imaging, and photothermal effect in vitro. a Cytotoxicity of PEG-Au@SiNRs. b LSCM images of CT-26 and 4T1 cells after incubation with RGD-Au@SiNRs (blocking with free peptides or not) or Au@SiNRs for $2 \mathrm{~h}$ at $37{ }^{\circ} \mathrm{C}$. Scale bars, $25 \mu \mathrm{m}$. c Cell viability of CT-26 cells, which were first incubated with RGD-Au@SiNRs or Au@SiNRs for $4 \mathrm{~h}$ and then irradiated by an 808 -nm laser $\left(0.8 \mathrm{~W} \mathrm{~cm}^{-2}\right)$ for $5 \mathrm{~min}$ as mean $\pm \mathrm{SD}(n=3)$. Asterisk $(* *)$ indicates $p<0.01$ 
(a)

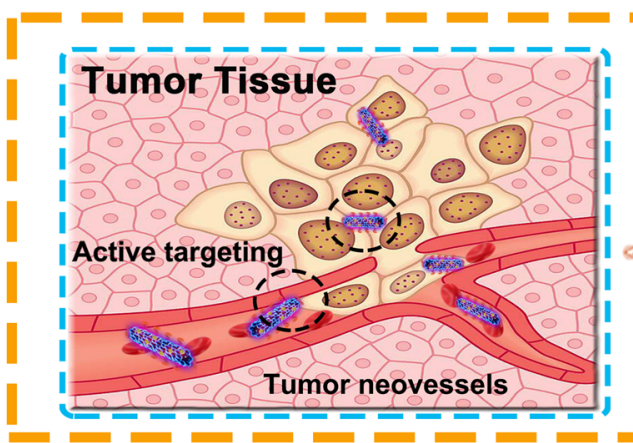

(b)

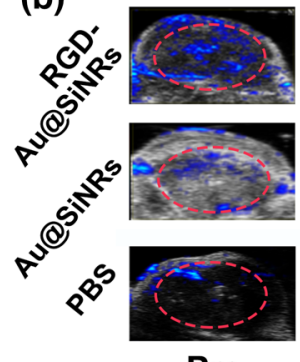

(d)

Pre

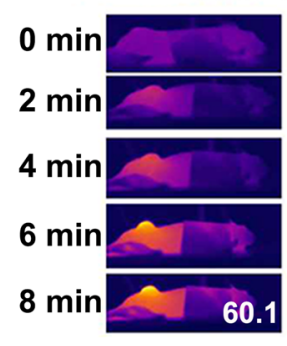

RGD-Au@SiNRs Au@SiNRs

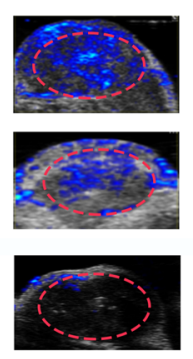

$12 \mathrm{~h}$

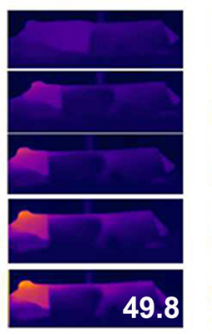

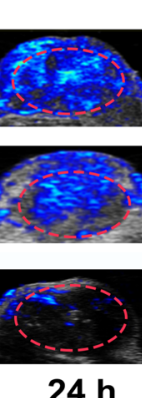

$24 \mathrm{~h}$

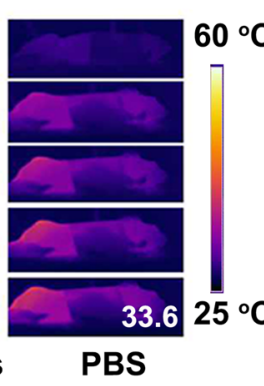

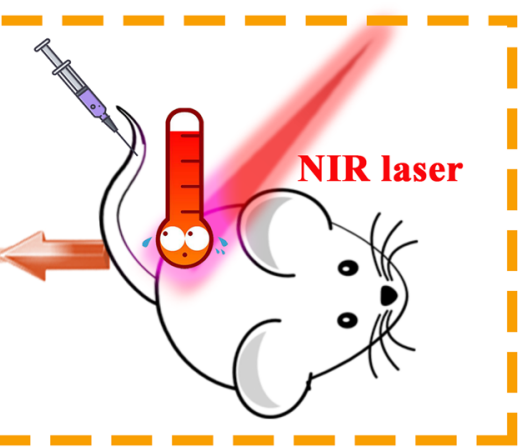

(c)

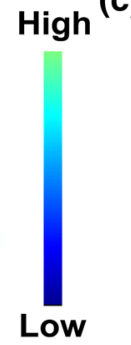

(e)
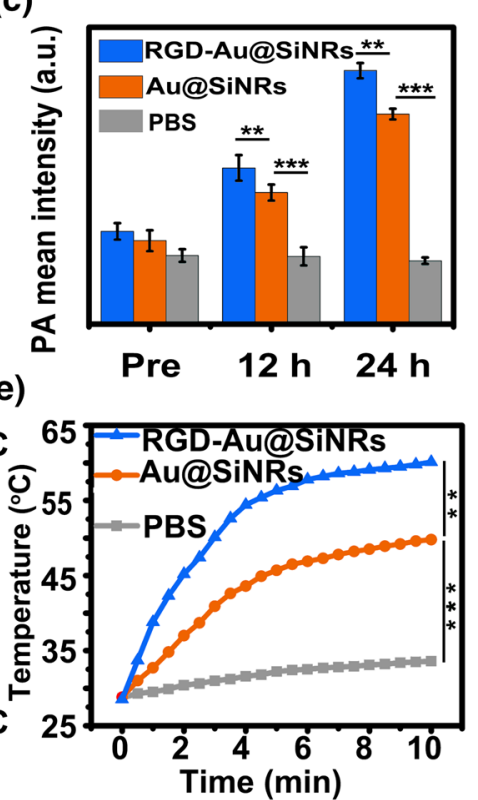

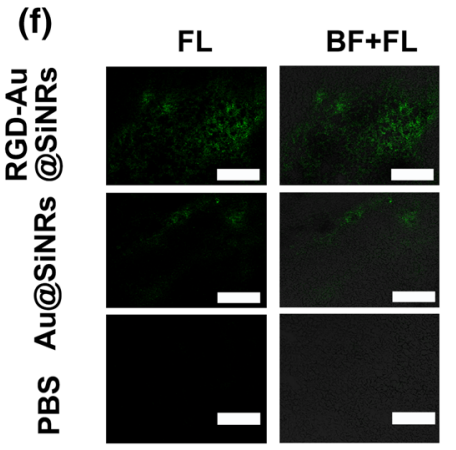

(g)

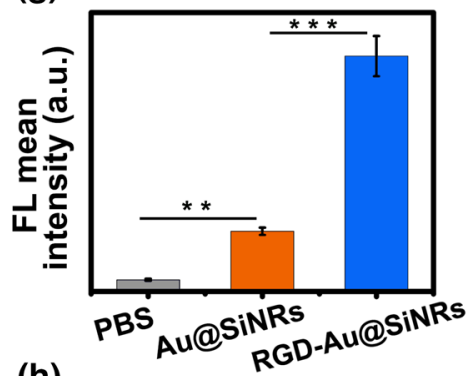

(h)

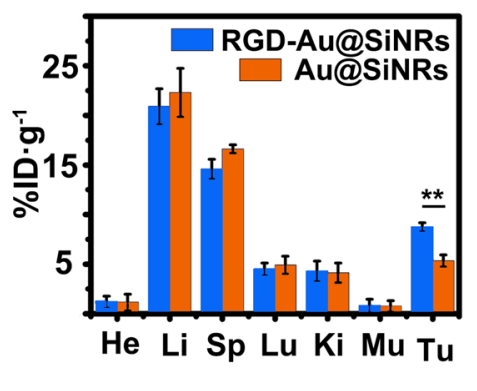

Fig. 4 Tumor-targeted multimodal imaging in vivo. a Schematic illustration of the active targeting of RGD-Au@SiNRs. b PA imaging and c the corresponding PA signal intensity of tumor regions of CT-26 tumor-bearing mice untreated and treated with RGD-Au@SiNRs, Au@SiNRs, or PBS for 12 and $24 \mathrm{~h}$. d Infrared thermal mapping images, and e corresponding temperature change of tumor regions of CT-26 tumor-bearing mice irradiated with an $808-\mathrm{nm}$ laser $\left(0.8 \mathrm{~W} \mathrm{~cm}^{-2}\right)$ for different times $(0-8 \mathrm{~min}$, time interval: $30 \mathrm{~s})$ at $24 \mathrm{~h}$ post-administration with RGD-Au@ SiNRs, Au@SiNRs, or PBS. f LSCM images of tumor sections at 24 h post-injection of PBS, Au@SiNRs, or RGD-Au@SiNRs. Scale bars, $100 \mu \mathrm{m}$, and $\mathbf{g}$ corresponding quantitative analysis of the fluorescence intensity. h The bio-distribution of RGD-Au@SiNRs and Au@SiNRs measured by ICP-OES at $24 \mathrm{~h}$ post-administration. Asterisk $(* *)$ indicates $p<0.01 ;(* * *)$ means $p<0.001$ 
(a)

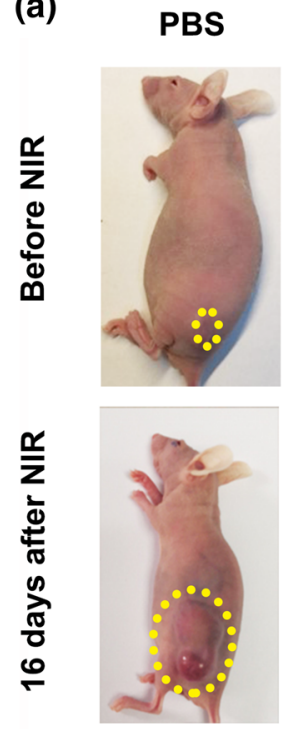

(d)

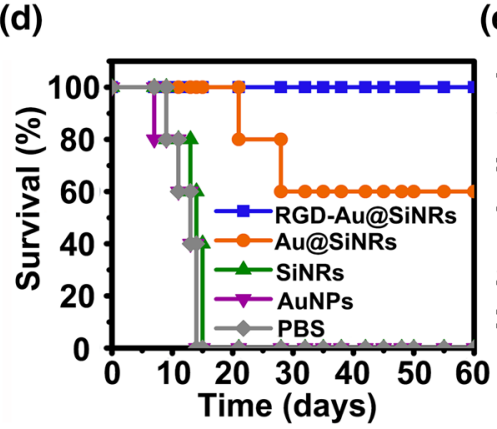

AuNPs
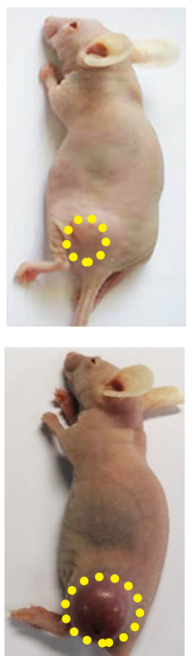

(e)
SiNRs
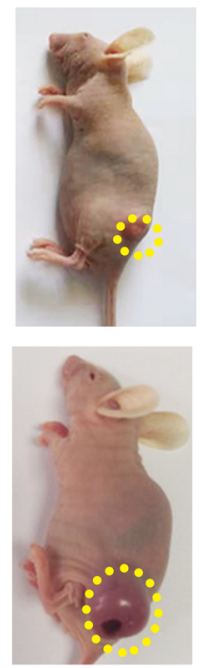

(e)
Au@SiNRs RGD-Au@SiNRs (b)
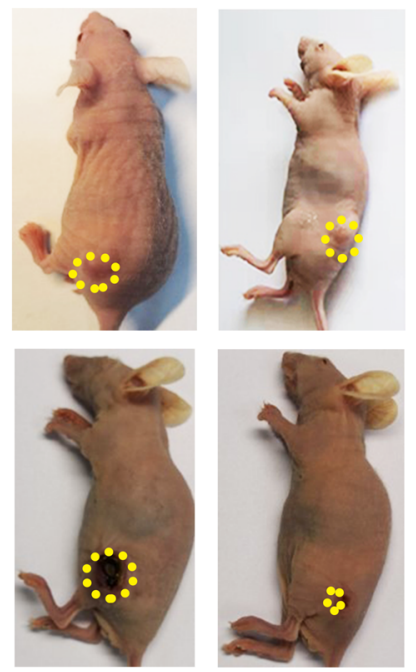

(f)
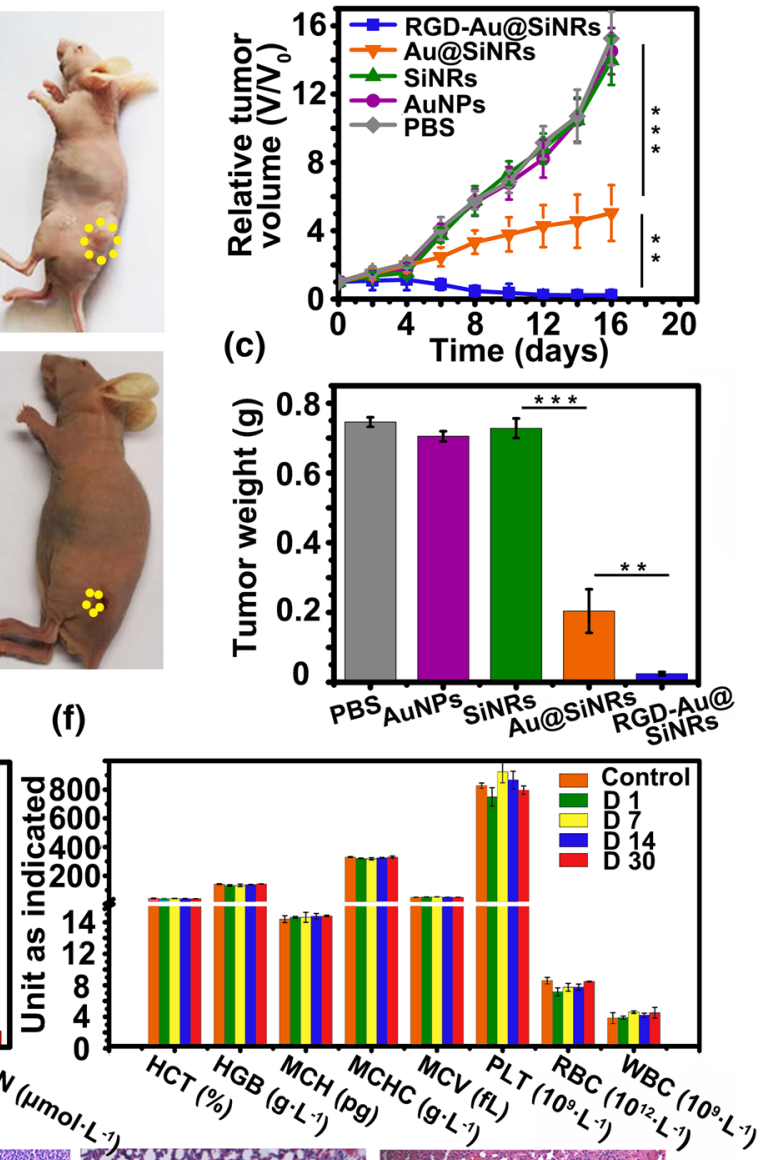

(g)
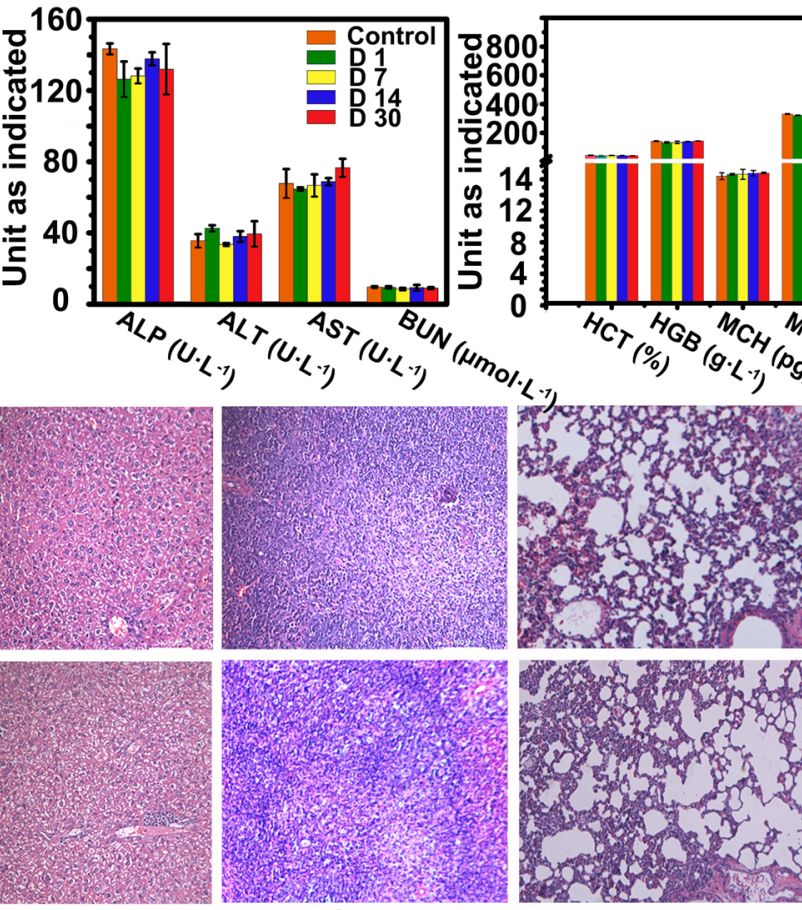

Lung

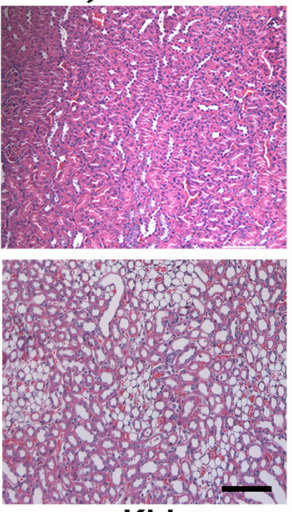

Spleen

Kidney

Fig. 5 Photothermal therapy and safety assessment. a Photographs of representative mice before and after the treatment with different agents and NIR irradiation. b Growth curves of tumor volumes of mice groups with NIR irradiation. c Weight of the excised tumors from the PTTtreated mice. d Survival curves of PTT-treated mice. e Serum biochemistry data including alkaline phosphatase, alanine aminotransferase, and aspartate aminotransferase, and blood urea nitrogen levels of control and RGD-Au@SiNRs-treated healthy mice. f Complete blood counts: hematocrit, hemoglobin, mean corpuscular hemoglobin, mean corpuscular hemoglobin concentration, mean corpuscular volume, blood platelets, red blood cells, blood levels of white blood cells, and platelets of control and RGD-Au@SiNRs-treated healthy mice. g H\&E staining of various organ tissues harvested from tumor-bearing mice at the end of treatment. Asterisk $(* *)$ indicates $p<0.01 ;(* * *)$ means $p<0.001$ 